Al-Azhar Bull. Sci. Vol. 24, No. 2 (Dec.): pp. 141-148, 2013.

\title{
POTENTIOMETRIC AND THERMODYNAMIC INVESTIGATION OF RHENIUM(V) COMPLEXES WITH 1-METHYL-2- MERCAPTOIMIDAZOLE
}

G. A. GOUDA, M. R. SHATAT, T. A. SEAF ELNASR AND M. H. ABDALLAH

Faculty of Science, Al-Azhar University, Assiut, 71524, Egypt

Corresponding author. E-mail: ggouda1973@yahoo.com

\section{Abstract}

Stability constants of 1-methyl-2-mercaptoimidazole with rhenium(V) were determined potentiometrically in acidic medium $(4 \mathrm{M} \mathrm{HCl})$ at different temperatures. The dissociation constants $p K$ of 1-methyl-2-mercaptoimidazole as well as the stability constants $(\log K)$ of their complexes were determined at varieties temperatures. The corresponding thermodynamic parameters $\left(\Delta G^{\mathrm{o}}, \Delta H^{\mathrm{o}}\right.$ and $\left.\Delta S^{\circ}\right)$ were determined and discussed. The formation of the metal complexes has been found to be exothermic.

Key words: Potentiometric, rhenium(V), formation constants, thermodynamics.

Characteristic stability constants may be important for predicting various chemical processes, such as isolation, extraction and concentration methods, ${ }^{[1,2]}$ since many elements are present in trace amounts, and can be separated by complexion reagents. Bejerrum's ${ }^{[3]}$ dissertation being initiative in developing this field. Metal complexation not only brings the reacting molecules together to give activated complex ${ }^{[4]}$ but also polarized electrons from the ligands towards the metal. The relation between stability and basicity of the ligands is indicated by the formation constant and free energy change values. The stability constant dependent on several factors such as: electronegativity, hardness or softness of the donor atoms on the ligand, the metal ion, nature of the ligand, the ionic radius and charge of the oxidation state on the metal core respectively. One available method is the potentiometric titration using ligand redox electrodes based on sulfur compounds and their oxidized forms. Many workers ${ }^{[5-18]}$ have reported their results on metalligand stability constants and their oxidized forms.

\section{Experimental}

To determine the formation constants of rhenium(V)-1-methyl-2mercaptoimidazole in $4 \mathrm{M} \mathrm{HCl}$ at different temperatures a potentiometric method of employed. Equilibrium concentrations of 1-methyl-2-mercaptoimidazole are calculated by the following equation:

$$
\lg [L]=\frac{E_{\text {initial }}-E_{i}}{1.9837 \cdot 10^{-4} T}+\lg C_{L}^{\text {initial }}+\frac{1}{2} \lg \frac{V_{\text {initial }}}{V_{\text {all }}}
$$

where $\mathrm{E}_{\text {initial }}$ - initial equilibrium potential of the oxidation-reduction system in the absence of rhenium $(\mathrm{V}) ; \mathrm{E}_{\mathrm{i}}$ - equilibrium potential at end point of titration. $C_{L}^{\text {initial }}$ - 
initial analytical concentration of 1-methyl-2-mercaptoimidazole; $V_{\text {initial }} / \mathrm{V}_{\text {all }}$ - the ratio of the initial volume to the total volume of the system: $T$ - the temperature of the experiment in Kelvin degree. Determined at each point of the titration equilibrium concentration of the ligand. The function $\bar{n}$ was calculated at each titration by the formula:

$$
\bar{n}=\frac{C_{L}-[L]}{C_{\operatorname{Re}(V)}}
$$

where $\bar{n}$ is the average degree of formation derived from the titration curves of a ligand with metals; $C_{L}$ is the concentration of 1-methyl-2-mercaptoimidazole; $[L]$ equilibrium concentration of 1-methyl-2-mercaptoimidazole; $C_{R e(V)^{-}}$the concentration of rhenium(V). All the calculations are employed using a computer «ntel Core $^{\mathrm{TM}} i 7$ ».

\section{Results and Discussion}

Among the ligand redox electrodes used for the study of complexes represent an important type on the basis of sulfur-containing organic compounds and their oxidized forms. The preparation of such electrodes is based on the reversible oxidation of thione or thiol-containing compounds to the corresponding disulfides.

The complexation process of rhenium(V) with 1-methyl-2-mercaptoimidazole proceeds stepwise and reversible. It is natural that the stability of these complexes depends on the nature of the substituent in the 1-methyl-2-mercaptoimidazole.

The addition of $\mathrm{H}_{2}\left[\mathrm{ReOCl}_{5}\right]$ to a solution $(0.0759 \mathrm{M})$ containing 1-methyl-2mercaptoimidazole $(0.0259 \mathrm{M})$ and its oxidized form in the acid medium $(4 \mathrm{M} \mathrm{HCl})$ causes a change in color of solution to red, with increasing concentration of $\mathrm{H}_{2}\left[\mathrm{ReOCl}_{5}\right]$ the solution is changed to purple, blue and finally to green. The adding, to the green solution 1-methyl-2-mercaptoimidazole color change of the solution is reversed. This fact indicates the gradation and reversibility of the complexation of rhenium(V) with 1-methyl-2-mercaptoimidazole. In the titration of rhenium(V)-1methyl-2-mercaptoimidazole system and its oxidized form, an increase in the magnitude of the equilibrium potential, indicating a other participation in complexation of rhenium(V) with 1-methyl-2-mercaptoimidazole than its oxidized form. At each point, equilibrium is established within 5-10 minutes. By potentiometric titration each values of the equilibrium concentration of 1-methyl-2mercaptoimidazole and $\mathrm{E}$ are determined. Using values of $[L]$ and taking together with both the analytical concentrations of $\mathrm{H}_{2}\left[\mathrm{ReOCl}_{5}\right]$ and 1-methyl-2mercaptoimidazole formation constants can be calculated. Some data determined by potentiometric titration of rhenium(V) with 1-methyl-2-mercaptoimidazole in $4 \mathrm{M}$ $\mathrm{HCl}$ at $273{ }^{\circ} \mathrm{K}$, are presented in Table 1. 
Table 1. Data obtained by potentiometric titration of rhenium(V) with 1-methyl-2mercaptoimidazole in $4 \mathrm{M} \mathrm{HCl}$ at $273{ }^{\circ} \mathrm{K}$

\begin{tabular}{|c|c|c|c|c|}
\hline$C_{\operatorname{Re}(V)} \cdot 10^{3}$ & $C_{L} \cdot 10^{2}$ & \multirow[t]{2}{*}{$\Delta E, m V$} & \multirow[t]{2}{*}{$-\log [L]$} & \multirow[t]{2}{*}{$\bar{n}$} \\
\hline \multicolumn{2}{|c|}{ Mole/l } & & & \\
\hline 4.536 & 2.323 & 61.54 & 2.745 & 4.726 \\
\hline 4.773 & 2.309 & 74.54 & 2.986 & 4.621 \\
\hline 5.240 & 2.281 & 108.98 & 3.624 & 4.308 \\
\hline 5.808 & 2.247 & 155.54 & 4.487 & 3.864 \\
\hline 6.358 & 2.214 & 178.98 & 4.923 & 3.481 \\
\hline 6.892 & 2.182 & 185.21 & 5.041 & 3.165 \\
\hline 7.413 & 2.151 & 188.32 & 5.101 & 2.901 \\
\hline 7.917 & 2.121 & 193.21 & 5.195 & 2.678 \\
\hline 8.409 & 2.092 & 196.47 & 5.258 & 2.487 \\
\hline 8.886 & 2.063 & 199.14 & 5.310 & 2.321 \\
\hline 9.351 & 2.035 & 201.44 & 5.355 & 2.176 \\
\hline 10.245 & 1.982 & 203.35 & 5.396 & 1.934 \\
\hline 11.092 & 1.931 & 207.24 & 5.474 & 1.741 \\
\hline 12.664 & 1.837 & 215.57 & 5.638 & 1.451 \\
\hline 14.089 & 1.752 & 220.54 & 5.740 & 1.243 \\
\hline 14.754 & 1.712 & 225.32 & 5.834 & 1.160 \\
\hline 15.996 & 1.638 & 228.62 & 5.904 & 1.024 \\
\hline 17.135 & 1.570 & 230.87 & 5.955 & 0.916 \\
\hline 18.184 & 1.508 & 233.36 & 6.010 & 0.829 \\
\hline 19.608 & 1.422 & 236.56 & 6.081 & 0.725 \\
\hline 20.881 & 1.346 & 238.47 & 6.128 & 0.644 \\
\hline 22.024 & 1.278 & 240.21 & 6.172 & 0.580 \\
\hline 23.692 & 1.178 & 244.55 & 6.270 & 0.497 \\
\hline 25.118 & 1.093 & 246.08 & 6.314 & 0.435 \\
\hline
\end{tabular}

Figure (1) showed potentiometric titration formation curves of rhenium(V)-1-methyl-2mercaptoimidazole complexes at different temperature in $4 \mathrm{M} \mathrm{HCl}$.

Potentiometric titration curves showed that rhenium(V)-1-methyl-2mercaptoimidazole system in presence of $4 \mathrm{M} \mathrm{HCl}$ at different temperatures consistently produced four types of complexes. The $\log K_{i}$ values of rhenium(V) with 1-methyl-2-mercaptoimidazole from the titration curves by Bjerrum method at half-integer values ${ }^{[19-20]}$ of the degree of formation $(\bar{n})$ is presented in Table 2 . 


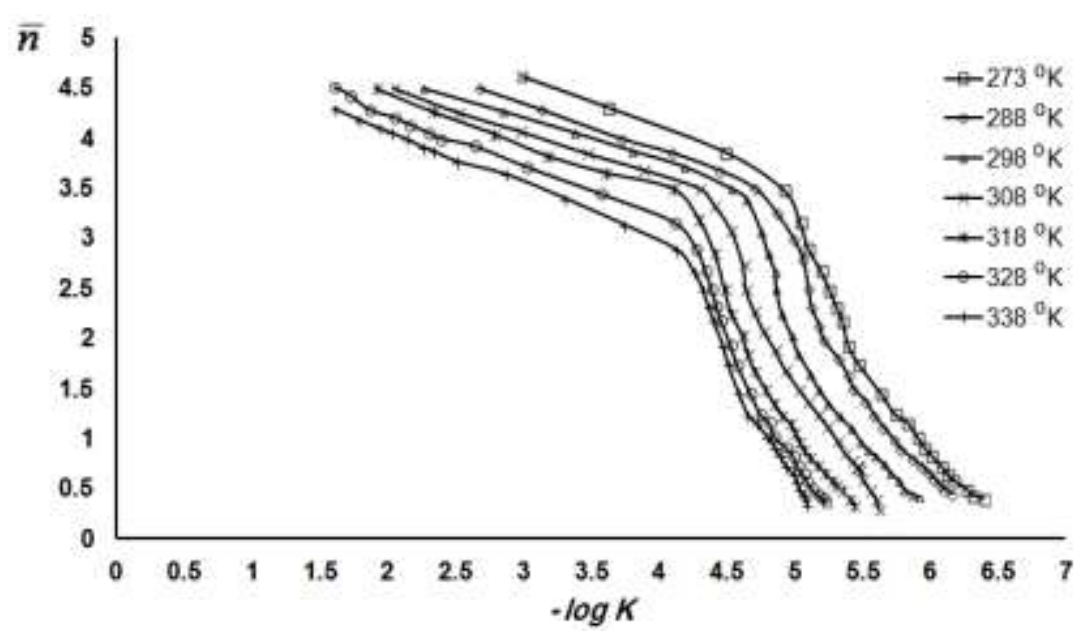

Fig. 1. Plots of $\bar{n}$ against (-log $K$ ) for rhenium(V) with 1-methyl-2-mercaptoimidazole complexes in $4 \mathrm{M} \mathrm{HCl}$ at different temperatures.

Table 2. Formation constant values of rhenium(V)-1-methyl-2-mercaptoimidazole in 4 $\mathrm{M} \mathrm{HCl}$ at different temperatures

\begin{tabular}{|c|c|c|c|c|}
\hline \multirow{2}{*}{$\mathrm{T},{ }^{\circ} \mathrm{K}$} & {$\left[\mathrm{ReOLCl}_{4}\right]^{-}$} & {$\left[\mathrm{ReOL}_{2} \mathrm{Cl}_{3}\right]$} & {$\left[\mathrm{ReOL}_{3} \mathrm{Cl}_{2}\right]^{+}$} & {$\left[\mathrm{ReOL}_{4} \mathrm{Cl}\right]^{2+}$} \\
\cline { 2 - 5 } & $\log K_{I}$ & $\log K_{2}$ & $\log K_{3}$ & $\log K_{4}$ \\
273 & 6.27 & 5.61 & 5.26 & 4.92 \\
288 & 6.10 & 5.43 & 5.10 & 4.71 \\
298 & 5.81 & 5.17 & 4.86 & 4.53 \\
308 & 5.55 & 5.03 & 4.64 & 4.31 \\
318 & 5.31 & 4.78 & 4.48 & 4.10 \\
328 & 5.13 & 4.65 & 4.39 & 3.45 \\
338 & 5.02 & 4.57 & 4.32 & 3.11 \\
\hline
\end{tabular}

These data show that with increasing amount coordinated molecules 1-methyl-2mercaptoimidazole $\log K_{i}$ decreases. Stepwise formation constant ratios were as follows: $K_{1} / K_{2}=4.57 ; K_{2} / K_{3}=2.24 ; K_{3} / K_{4}=2.19$ at $273{ }^{\circ} \mathrm{K}$. These data indicate that the ratio stepwise formation constants are not so large enough, so it was necessary to clarify the estimated constants either successive approximation method or by the "pH-meter» program ${ }^{[21]}$. However, attempts to refine the estimated formation constants were not sufficient.

The equilibrium constant $(\mathrm{K})$ varies with temperature according to the Van't Hoff $^{[22-23]}$ equation:

$$
\frac{d \ln K}{d T}=\frac{\Delta H^{o}}{R T^{2}}
$$


$R$ is the universal gas constant, $\mathrm{T}$ is the absolute temperature (in ${ }^{\circ} \mathrm{K}$ ) and $\Delta H^{\mathrm{o}}$ is the enthalpy change. To obtain the integrated equation, it is convenient to first rewrite the Van't Hoff equation as

$$
\frac{d \ln K}{d \frac{1}{T}}=-\frac{\Delta H^{o}}{R}
$$

Thus, for exothermic reactions, the $\Delta H^{0}$ is negative and $K$ decreases with temperature, but for endothermic reactions $\Delta H^{\mathrm{o}}$ is positive and $K$ increases with temperature. In accordance with the data ${ }^{[24]}$ values of stability constants after verifying these methods vary slightly. In this regard, the stability constant of rhenium(V)-1-methyl-2-mercaptoimidazole complexes, some of the potentiometric titration curves, were used to estimate the thermodynamic properties of the complexation by the temperature coefficient. The $\Delta H^{\mathrm{o}}$ values were determined frome the slope of the straight line obtained by plotting $\log K_{i}$ against $1 / T$ (Fig. 2). The change in entropy is determined by the interval intercepts on the y-axis, these lines $\left(\Delta S^{0}=R * \text { interception }\right)^{[25,26]}$. Gibbs energy ${ }^{[27]}$ was calculated from the equation $\Delta G^{\mathrm{o}}=\Delta H^{\mathrm{o}}-T \Delta S^{\mathrm{O}}$ (Table 3). Calculated thermodynamic function showed that the isobaric-isothermal capacity becomes less negative with increasing number of coordinated molecules of 1-methyl-2-mercaptoimidazole. This experimental finding may be due to an increase in the steric hindrance that prevent in entering molecules of 1-methyl-2-mercaptoimidazole to center the inner sphere complexes [28].

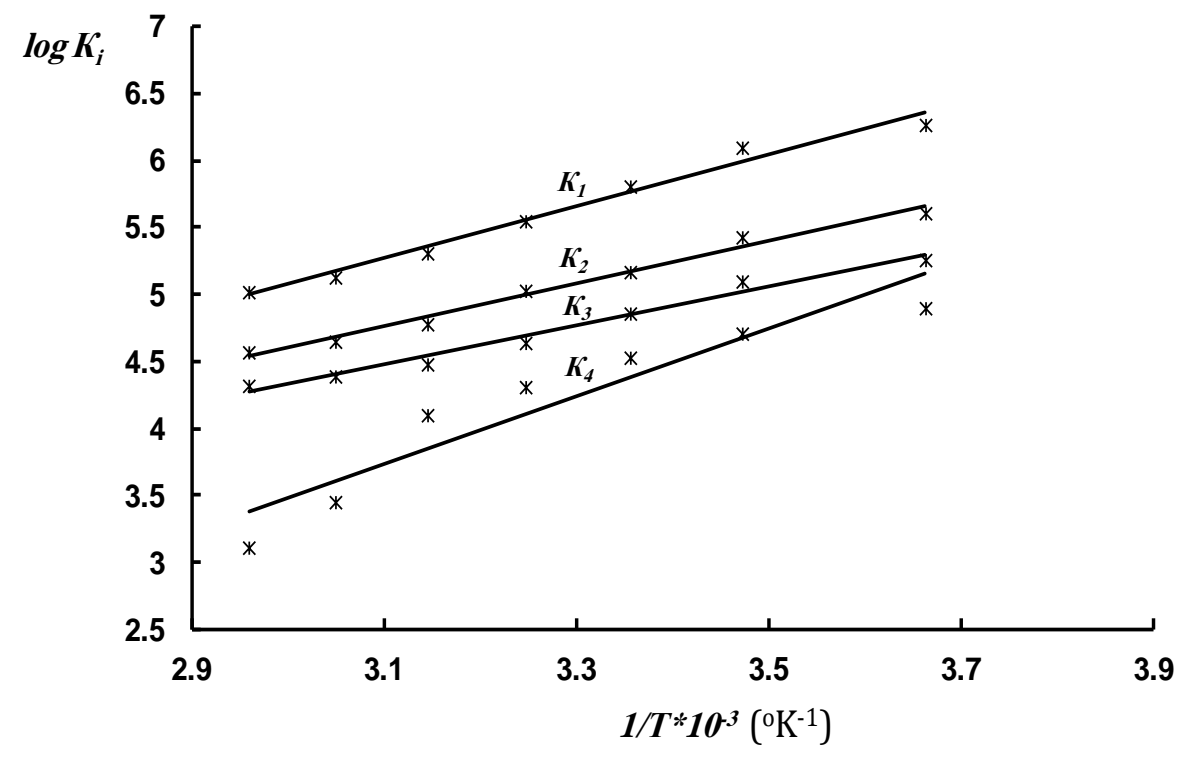

Fig. 2. Plots of $\log K_{i}$ against $1 / T$ for rhenium(V)-1-methyl-2-mercaptoimidazole complexes in $4 \mathrm{M} \mathrm{HCl}$ at different temperatures. 
Table 3. The thermodynamic parameters of the formation rhenium(V)-1-methyl-2mercaptoimidazole complexes in $4 \mathrm{M} \mathrm{HCl}$ at different temperatures

\begin{tabular}{|c|c|c|c|}
\hline Species & $-\Delta H^{\mathrm{o}}, \mathrm{kJ} / \mathrm{mole}$ & $-\Delta G^{\mathrm{o}}, \mathrm{kJ} / \mathrm{mole}$ & $-\Delta S^{\mathrm{o}}, \mathrm{J} / \mathrm{mole} \cdot \mathrm{K}$ \\
\hline$\left[\mathrm{ReOLCl}_{4}\right]^{-}$ & 36.97 & 32.88 & 13.70 \\
{$\left[\mathrm{ReOL}_{2} \mathrm{Cl}_{3}\right]$} & 22.36 & 28.97 & 3.05 \\
{$\left[\mathrm{ReOL}_{3} \mathrm{Cl}_{2}\right]^{+}$} & 27.84 & 27.65 & 0.65 \\
{$\left[\mathrm{ReOL}_{4} \mathrm{Cl}^{2+}\right.$} & 48.78 & 24.99 & 79.86 \\
\hline
\end{tabular}

The values of the entropy changes for the mono-substituted complex compared to the disubstituted complex has a much greater significance ${ }^{[29]}$. Thus, as the higher value of $\Delta S^{\circ}$ can be interpreted in favor substitution of chloride ion in the transposition to the oxygen oxorhenium groups is apparently due to the fact that $\mathrm{Re}-\mathrm{Cl}$ distance being in trans-position to the oxygen of the oxorhenium group in the equatorial plane ${ }^{[30]}$. Significant decrease of $\Delta S^{\circ}$ is probably due to the fact that the introduction of a second molecule of 1-methyl-2-mercaptoimidazole in the inner coordination sphere becomes more difficult and that such molecule may replace one of the four chloride ions that are in the equatorial plane ${ }^{[31]}$.

Mole fractions are commonly used to calculate the concentrations of the individual complexes on the basis of the formation constants. Mole fractions of a particular form of the complex compressed as ratio of the concentration of the complex to the total concentration of the metal ion $\left(\left[M L_{i}\right] /\left[\right.\right.$ Metal ion] $\left.=X_{i}\right)$. To determine the field dominance of a complex in the form of rhenium(V)-1-methyl-2mercaptoimidazole in $4 \mathrm{M} \mathrm{HCl}$ was calculated from the distribution curves at different temperatures. Figure 3 shows the distribution curves of complex models at $328^{\circ} \mathrm{K}$.

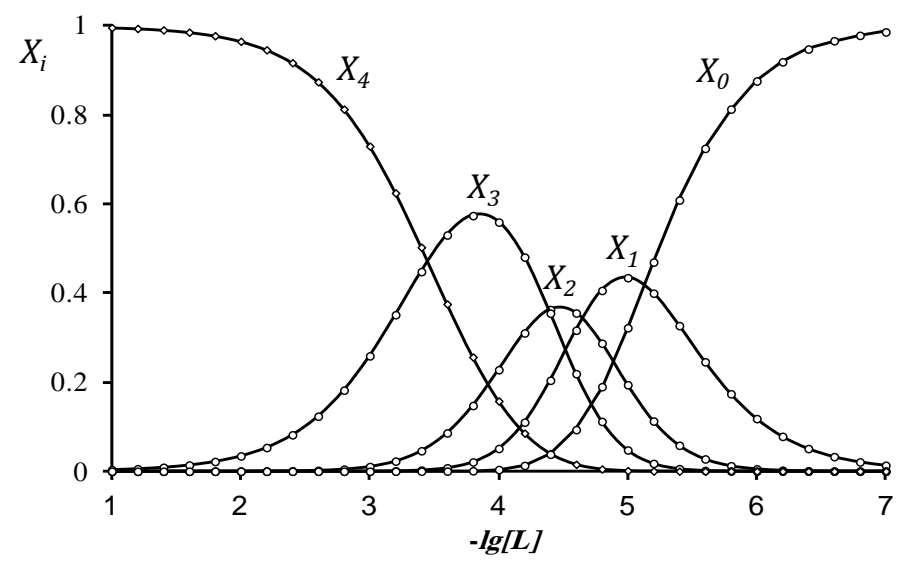

Fig. 3. Distribution curves of rhenium(V)-1-methyl-2-mercaptoimidazole complexes at $328^{\circ} \mathrm{K}$; where $X_{0}=\left[\operatorname{ReOCl}_{5}\right]^{2-}, X_{1}=\left[\operatorname{ReOLCl}_{4}\right]^{\top}, X_{2}=\left[\operatorname{ReOL}_{2} \mathrm{Cl}_{3}\right], X_{3}=$ $\left[\operatorname{ReOL}_{3} \mathrm{Cl}_{2}\right]^{+}, X_{4}=\left[\mathrm{ReOL}_{4} \mathrm{Cl}_{2}\right]^{2+}$. 
Analysis of the distribution curves show that increasing temperature has little effect on the proportions of the maximum output value for all complexes. Increasing temperature causes $X_{i}^{\max }$ being shifted towards higher values of equilibrium concentration of 1-methyl-2-mercaptoimidazole (Table 4).

Table 4. The $X_{i}^{\max }$ output equilibrium values for rhenium(V)-1-methyl-2 mercaptoimidazole complexes in $4 \mathrm{M} \mathrm{HCl}$ at different temperatures

\begin{tabular}{|c|c|c|c|c|c|c|c|}
\hline \multirow{2}{*}{ Species } & \multicolumn{7}{|c|}{ Values $\lg [L]$ at $X_{i}^{\max }$} \\
\cline { 2 - 8 } & $273{ }^{\circ} \mathrm{K}$ & $288{ }^{\circ} \mathrm{K}$ & $298{ }^{\circ} \mathrm{K}$ & $308{ }^{\circ} \mathrm{K}$ & $318{ }^{\circ} \mathrm{K}$ & $328{ }^{\circ} \mathrm{K}$ & $338{ }^{\circ} \mathrm{K}$ \\
\hline$\left[\mathrm{ReOLCl}_{4}\right]^{-}$ & 6.0 & 5.8 & 5.6 & 5.4 & 5.2 & 5.0 & 4.8 \\
{$\left[\mathrm{ReOL}_{2} \mathrm{Cl}_{3}\right]$} & 5.4 & 5.2 & 5.0 & 4.8 & 4.6 & 4.4 & 4.4 \\
{$\left[\mathrm{ReOL}_{3} \mathrm{Cl}_{2}\right]^{+}$} & 5.0 & 4.8 & 4.6 & 4.4 & 4.2 & 3.8 & 3.6 \\
\hline
\end{tabular}

Based on these data it is possible to choose the optimum conditions for the isolation of certain complex, establishing their composition and structure.

\section{References}

1. Andres Garcia E. and Blanco Gomis D., Microchem. Acta, 1996, 4, 124.

2. Cao S. and Zhang M., J. Trace Microprobe Tech., 1999, 17, 157.

3. Bjerrum J., Metal amine formation in aqueous solutions: theory of the reversible step reactions, Haase P. and Sons (Copenhagen, Denmark), 1941, 296.

4. Florence A.T. and Attwood D., Physical principle's of pharmacy, Macmillan (London), 1981.

5. Poddar S.N., Dey K. and Poddar N.G., Indian J. Chem., 1970, 8, 364.

6. Schwarzenbach G. and Ackerman H., Helv. Chim. Acta, 1948, 31, 1029.

7. Pund D.A., Bhagwatkar R.A., Tayade D.T. and Rathod D.B., Rasayan J. Chem., 2010, 3(2), 246.

8. Tihile M.S., Journal of Chemical and Pharmaceutical Research, 2012, 4(4), 2223.

9. Dipak T.T., International Journal of Chemistry, 2011, 3(1), 36.

10. Gudadhe S., Narwade M.L. and Jamode V.S., Acta Ciencia Indica (Chem.), 1985, 11, 234.

11. Saha N., Dalia M. and Sinha S., Indian J. Chem., 1986, 25A, 629.

12. Rana A.K. and Shah J.R., J. Indian Chem. Soc., 1986, 63, 281.

13. Fukuda V., Morishita R. and Sone K., Bull. Chem. Sep. Jpn., 1985, 49, 1017.

14. Jolly V.S., Arora G.D. and Taiwar P., J. Indian Chem. Soc., 1990, 61, 1001.

15. Natrajan C. and Thormaraj P., Indian J. Chem., 1991, 30A, 722.

16. Narwade M.L., Chincholkar M.M. and Sathe S.W., J. Indian Chem. Soc., 1985, 62, 194.

17. Sawalakhe P.D. and Narwade M.L., J. Indian Chem. Soc., 1995, 70, 25.

18. Kadu M.V. and Jamode V.S., Asian J. Chem., 1999, 11, 420. 
19. Bjerrum J. and Bang E., Acta Chem. Scand. Ser., 1979, A33, 297.

20. Irving H.M. and Rossotti H.S., J. Chem. Soc., 1953, 3397; J. Chem. Soc., 1954, 2904.

21. Valeria M. Nurchi, Guido Crisponi, Tiziana Pivetta, Martina Donatoni and Maurizio Remelli, J. of Inorg. Biochem., 2008, 102(4), 684.

22. Atkins, Peter; De Paula, Julio (10 March 2006). Physical Chemistry (8th ed.). Freeman W.H. and Company, p. 212.

23. Ives D.J.G., Chemical Thermodynamics, University Chemistry, Macdonald Technical and Scientific, 1971.

24. Rigano C., Rizzarelli E. and Sammartano S., Thermochim. Acta, 1979, 33, 211.

25. Abd Erbary H.M., Shehata H.A., El Arab M.A.F., Mohamed A.A. and Emara M.M., J. Ind. Chem. Soc., 1996, 73, 25.

26. Nair U.S.A. and Nancollas G.H., J. Chem. Soc., 1961, 255, 4367.

27. Dickerson R.E., Geis I. and Benjamin I.W.A., Chemistry, Matter and the Universe, (USA), 1976.

28. Amindzhanov A.A. and Kurbanov N.M., Journal of Inorganic Chemistry (Russian Federation ), 1990, 35(3), p. 672-678.

29. Promila D.T. and Lonibala R., J. Chem. Eng. Data, 2010, 55, 1166.

30. Amindzhanov A.A. and Gagieva S.Ch., Journal of Inorganic Chemistry (Russian Federation ), 1996, 35(3), p. 1867-1871.

31. Amindzhanov A.A., Akhmedov K.U. and Kotegov K.V., Journal of Inorganic Chemistry (Russian Federation ), 1988, 33(2), p. 379-384. 